OPEN ACCESS

Edited by:

Francesco Fazio,

Albert Einstein College of Medicine,

United States

Reviewed by:

Ravindra M. Samartha,

Bhopal Memorial Hospital \& Research

Centre, India

Are Hugo Pripp,

Oslo University Hospital, Norway

*Correspondence:

Gang Zhu

zhugang6666@126.com

${ }^{\dagger}$ These authors have contributed equally to this work

Specialty section: This article was submitted to

Neuropharmacology,

a section of the journal

Frontiers in Neuroscience

Received: 08 January 2020 Accepted: 22 April 2020

Published: 12 June 2020

Citation:

Wei L, Lin C, Zhong $M$, Zhang J and Zhu G (2020) Hemoglobin Concentration May Affect the Effect of Atorvastin on Chronic Subdural Hematoma After Burr-Hole Drainage at High Altitude.

Front. Neurosci. 14:503. doi: 10.3389/fnins.2020.00503

\section{Hemoglobin Concentration May Affect the Effect of Atorvastin on Chronic Subdural Hematoma After Burr-Hole Drainage at High Altitude}

\author{
Linjie Weit,2t, Chi Lin ${ }^{3 \dagger}$, Mingfeng Zhong ${ }^{4 \dagger}$, Jianbo Zhang ${ }^{5}$ and Gang Zhu ${ }^{1 *}$ \\ ${ }^{1}$ Department of Neurosurgery, Southwest Hospital, Third Military Medical University (Army Medical University), Chongqing, \\ China, ${ }^{2}$ Department of Neurosurgery, PLA 956th Hospital, Linzhi, China, ${ }^{3}$ Department of Neurosurgery, First People's \\ Hospital of Honghe City, Yunnan, China, ${ }^{4}$ Department of Function, The People's Hospital of Weiyuan County, Sichuan, \\ China, ${ }^{5}$ Department of Neurosurgery, The General Hospital of Southern Theater Command PLA, Guangzhou, China
}

Objective: Chronic subdural hematoma $(\mathrm{CSDH})$ is a common disease. Atorvastatin calcium can increase $\mathrm{CSDH}$ absorption. However, whether atorvastatin can increase hematoma absorption and reduce recurrence at high altitudes is not clear.

Methods: After burr-hole drainage, CSDH patients were divided into an atorvastatin group and a control group. Follow-up computed tomography (CT) was performed on day 1 , months 1,2 , and 3 after surgery. Then, the recurrence rate, poor therapeutic effect, time to recurrence, poor surgical result, recurrence with operation, CSDH volume, and Markwalder grading scale score (MGSS) were calculated, and related risk factors were analyzed.

Results: The non-recurrent and recurrent patients in the control group differed significantly in terms of the hemoglobin concentration (HB) [176.24 \pm 16.43 vs. $194.25 \pm 12.34$ (g/L), $p<0.01]$, CT value [41.92 \pm 10.76 vs. $34.12 \pm 8.78(\mathrm{Hu})$, $p<0.01$ ], and low-density time [3.88 \pm 1.04 vs. $5.50 \pm 0.87$ (d), $p<0.01$ ]. The non-recurrent and recurrent patients in the atorvastatin group differed significantly in terms of the HB [172.66 \pm 16.41 vs. $190.45 \pm 10.23$ (g/L), $p<0.01]$, CT value [38.91 \pm 7.16 vs. $29.50 \pm 8.61(\mathrm{Hu}), p<0.01]$, and mixed [2 vs. $4(\mathrm{n}), p<0.05]$ and low-density time [4.09 \pm 0.75 vs. $5.45 \pm 1.12$ (d), $p<0.01]$. The logistic regression analysis showed that $\mathrm{HB}$ [odds ratio, 1.14; 95\% confidence interval $(\mathrm{Cl}), 1.04-1.25$ in the control group, odds ratio, $1.13 ; 95 \% \mathrm{Cl}, 1.03-1.23$ in the atorvastatin group] and low-density time (odds ratio, 3.53; $95 \% \mathrm{Cl}, 1.42-8.74$ in the control group, odds ratio, 2.53; $95 \% \mathrm{Cl}, 1.10-5.80$ in the atorvastatin group) were possible risk factors for the two groups. The receiver operating characteristic curves showed that the area under the 
receiver operating characteristic curve values for the $\mathrm{HB}, \mathrm{CT}$ value $(\mathrm{Hu})$, and low-density time were $0.812,0.702$, and 0.755 for all subjects; 0.812, 0.719 , and 0.790 for the control group; and $0.807,0.682$, and 0.756 for the atorvastatin group, respectively. The postoperative follow-up results showed that there was no significant difference in the recurrence rate, poor therapeutic effect, time to recurrence, poor surgical result, recurrence with operation, CSDH volume, or MGSS between the two groups.

Conclusion: The effect of atorvastatin was not significant after the operation. The risk factors for $\mathrm{CSDH}$ recurrence were the HB and low-density time. The HB was the most specific and sensitive predictor of $\mathrm{CSDH}$ recurrence.

Keywords: high altitude, atorvastatin, chronic subdural hematoma, burr hole, hemoglobin concentration

\section{INTRODUCTION}

Chronic subdural hematoma (CSDH) is a common and frequently occurring disease, and the incidence rises with age (Sahyouni et al., 2017). Burr-hole drainage is a routine operation for the treatment of CSDH (Tang et al., 2018; Soleman et al., 2019; Khan et al., 2019), but the recurrence rate is $\sim 10$ 39\% (Kim et al., 2014; Jang et al., 2015). Local inflammatory responses may contribute to the recurrence of CSDH (Jaiswal and Sontakke, 2012; Zhang et al., 2020). Atorvastatin is an inhibitor of HMG-CoA reductase, and it can inhibit the biosynthesis of hydroxymethyl glutaryl coenzyme a reductase and cholesterol. In recent years, studies have shown that CSDH absorption significantly increases and that the recurrence rate is reduced with the use of atorvastatin (Tang et al., 2018; Quan et al., 2019; $\mathrm{He}$ et al., 2020). Many studies have investigated the effect of atorvastatin on CSDH in low-altitude regions, but few studies have been carried out in high-altitude regions. However, whether atorvastatin can increase hematoma absorption and reduce recurrence is unclear at high altitudes. In addition, high-altitude areas can lead to increased HB and coagulation dysfunction due to hypoxia (Xie et al., 2015). It is not clear whether this will affect CSDH absorption with atorvastatin calcium. Thus, the effect of atorvastatin treatment on CSDH was retrospectively summarized after burr-hole drainage at high altitudes.

\section{MATERIALS AND METHODS}

\section{Patient Selection}

This study was approved by the ethics committee of the Southwest Hospital. Patient consent was waived by our review board. Case data are retrospective studies. From February 2013 to December 2018, a total of 135 patients were enrolled who had CSDH as determined by computed tomography (CT) (GE Lightspeed 64, Tokyo, Japan) scans and related examinations. Thirty-eight patients were excluded according to the following criteria (Figure 1): surgical indication: (1) the displacement of the midline was more than $0.8 \mathrm{~cm}$; (2) the ipsilateral

Abbreviations: CT, computed tomography; CSDH, chronic subdural hematoma; $\mathrm{SD}$, standard deviation; ROC, receiver operating characteristic; Hu, hounsfield unit; MGSS, Markwalder grading scale score; HB, hemoglobin concentration. ventricle was obviously compressed or had disappeared; (3) the hematoma volume was more than $50 \mathrm{~mL}$; (4) if the head CT did not reveal one of the first three criteria, the Markwalder grading scale score (MGSS) was 1-2. A total of 97 patients were treated with burr-hole drainage. Those patients were divided into the atorvastatin group and the control group, depending on whether atorvastatin was administered after the operation. The relevant data of the two groups were collected and recorded in detail upon admission, including sex, age, trauma history, medication history, hemoglobin concentration (HB), hematoma location, hematoma density, hematoma size, neurological function, symptom improvement, and comorbidity. Considering the risk of severe CSDH with increased intracranial pressure and prognosis, according to the literature (Wang et al., 2014), grades 0-3 CSDH was included, but this study enrolled only patients with grades $0-2 \mathrm{CSDH}$ at high altitude. Figure $\mathbf{1}$ shows the steps for patient review. All data were independently and blindly reviewed by two senior neurosurgeons.

\section{Operation Method}

According to the CT of the head, the largest hematoma plane was selected as the positioning point for drilling. All patients were placed under local anesthesia, and a $20 \mathrm{~mm} \mathrm{YL-1} \mathrm{puncture}$ needle (Beijing Wan Tie Fu Medical Apparatus Co. Ltd., Beijing, China) was used for drilling after selecting the positioning point. The positioning point was strictly and slowly rinsed with warm normal saline until the rinsing fluid was clear (Figure 2). The follow-up head CT examination found obvious brain tissue expansion, subdural hematoma $\leq 50 \mathrm{~mL}$ in volume, and lowdensity CSDH [CT value $(\mathrm{Hu}) \leq 30$ : equal to $\mathrm{CT}$ value $(\mathrm{Hu})$ of hypodense hematoma] on days $2-6$ after the operation, and the drainage tube was removed (Figure 3). After 6 days, subdural hematoma was not $<50 \mathrm{~mL}$ in volume, and there was no lowdensity CSDH [CT value $(\mathrm{Hu}) \leq 30$ ]. The drainage tube was also pulled out, and the results were recorded as poor.

\section{Design and Atorvastatin Therapy}

There were 43 patients in the atorvastatin group and 54 in the control group. Patients were randomly assigned to two groups. The patients took a once-daily oral dose of $20 \mathrm{mg}$ atorvastatin for 3 months (Lipitor; Pfizer, Inc., Dalian, China) in the atorvastatin group. If the $\mathrm{CSDH}$ disappeared within 3 months, atorvastatin 


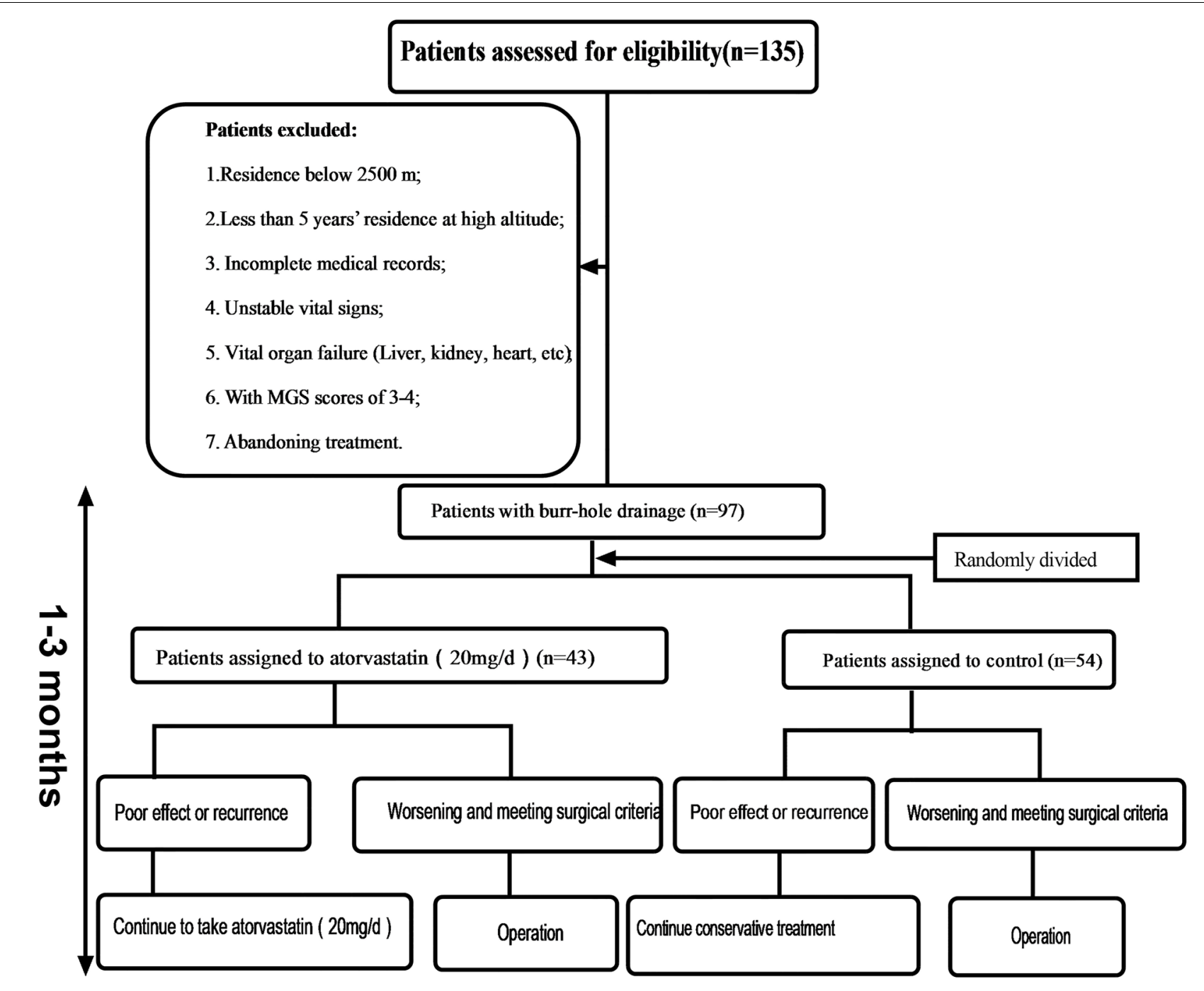

FIGURE 1 | A flowchart for the identification process of eligible patients.

was stopped. The patients were not given atorvastatin in the control group; in addition to atorvastatin, other treatments and drugs were the same in both groups. All patients were followed up regularly for 3 months.

\section{Evaluation and Follow-Up}

Each patient had a head CT scan to measure hematoma volume before and after the treatment. Hematoma volume was calculated based on ImageJ software (Bethesda, Maryland, USA) as previously described (Hanell et al., 2012). Chronic subdural hematoma was classified as mixed, hypodense, isodense, or hyperdense, according to the CT value ( $\mathrm{Hu}$ ) (Figure 4). If it was a mixed hematoma, the high-, medium-, and low-density hematoma CT values ( $\mathrm{Hu}$ ) were calculated and then averaged. The MGSS and Glasgow Coma Scale grades were obtained before the treatment (Table 1). The time for the $\mathrm{CSDH}$ to become low density was recorded after the operation (Figure 3). Follow-up CT was performed on the first day, months 1, 2, and 3 after the operation, and then the time to recurrence, recurrence with operation, poor therapeutic effect, poor surgical result, $\mathrm{CSDH}$ volume, and MGSS were calculated. If the patient's condition worsened, additional CT scans were performed. The definition of non-recurrence was CSDH volume $\leq 50 \mathrm{~mL}$, CT value $(\mathrm{Hu}) \leq 30$, and MGSS < 1: (1) poor effect: although the hematoma did not reach the upper recurrence standard, the absorption was poor [hematoma volume (third month) divided by hematoma volume (upon admission) $\geq 50 \%$ ]; (2) good effect: complete disappearance of hematoma or hematoma volume (third month) $<50 \%$. The definition of recurrence was $\mathrm{CSDH}$ volume $>50 \mathrm{~mL}$, CT value $(\mathrm{Hu})>30$, or symptoms of neurological dysfunction that appeared again (MGSS $\geq 1$ ) (Abouzari et al., 2007). If recurrent CSDH was consistent with surgical indications, surgical treatment was performed with a YL1 puncture needle. Logistic regression analysis of risk factors and receiver operating characteristic (ROC) curves were performed for non-recurrent and recurrent patients using the relevant 
A

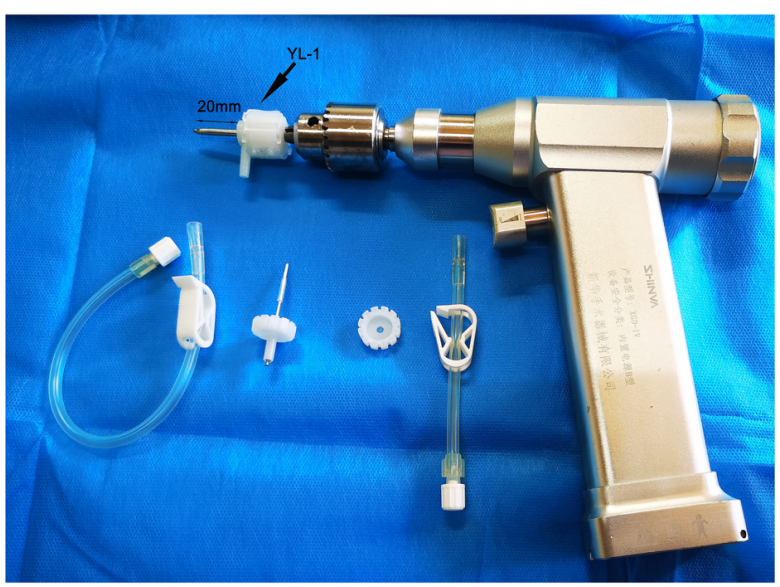

C

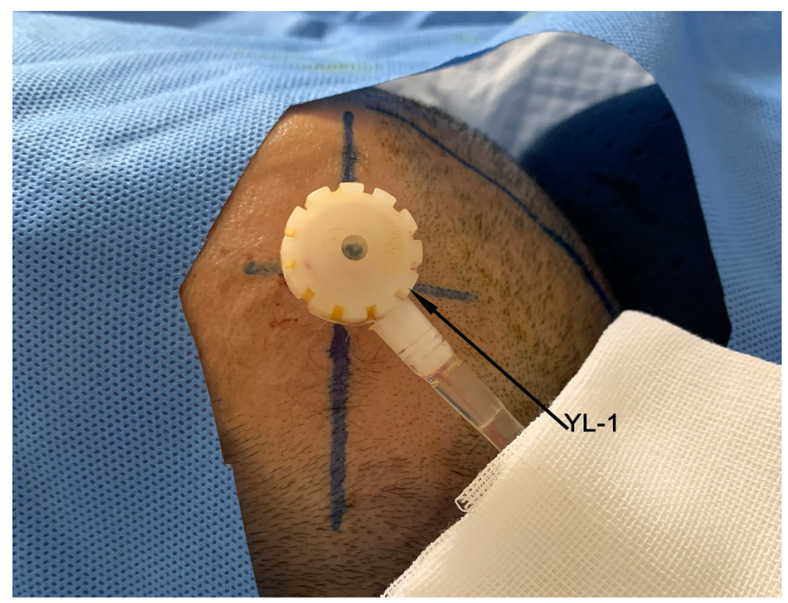

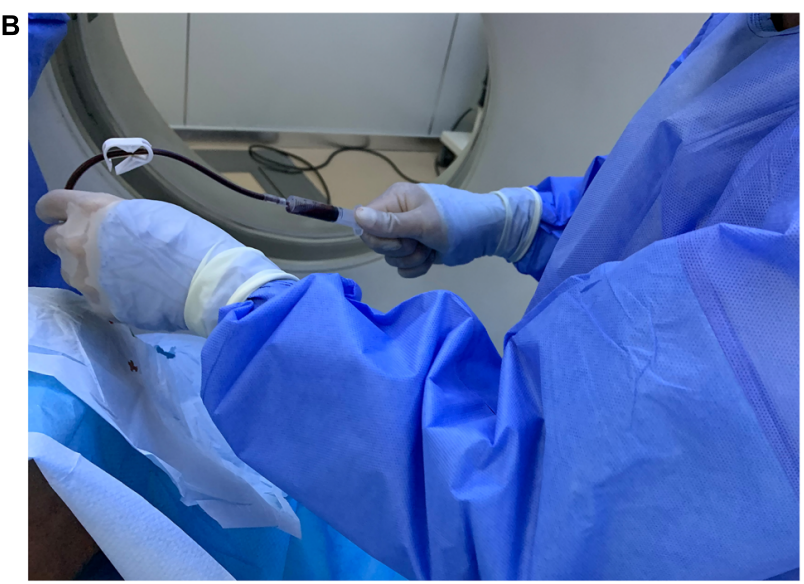

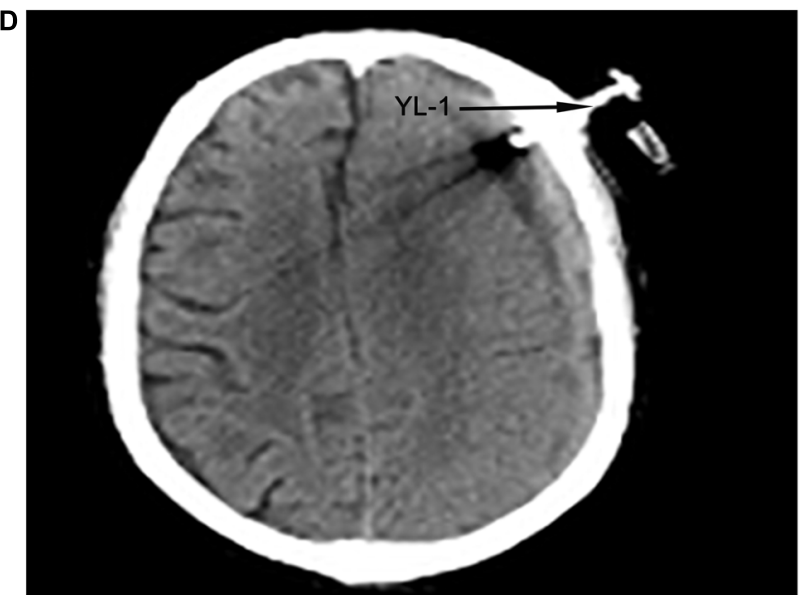

FIGURE 2 | Yl-1 drainage tube (black arrow) before surgery, drill length $20 \mathrm{~mm}$ (two black arrows) (A). YL-1 drainage tube was used to draw and rinse the CSDH during the operation (B). YL-1 drainage tube (black arrow) continued to drain the hematoma after the operation (C). YL-1 drainage tube (black arrow) on postoperative CT (D).

data upon admission. If the patient's recovery was poor or if there was hematoma recurrence, treatment was carried out as shown in Figure 1, but regardless of the outcome of the two groups of patients, the treatment effect was evaluated at the third month.

\section{Statistical Analysis}

All analyses were performed using SPSS 19 (IBM Corp., Armonk, NY, United States). Normally distributed data are expressed as the mean \pm standard deviation. Quantitative data were analyzed between the two groups using separate $t$-tests and analysis of variance, whereas categorical data were compared using $\chi^{2}$-tests between the atorvastatin and control groups. The risk factors for the atorvastatin and control groups were analyzed by a single factor. Logistic regression was used to analyze risk factors between the atorvastatin group and the control group. Receiver operating characteristic curve analysis was used to investigate the diagnostic relationship between risk factors and hematoma recurrence. The Youden index was used to determine the optimal cutoff value as previously described. Differences were considered significant at $P<0.05$.
All data analyses were conducted by two investigators in a blinded manner.

\section{RESULTS}

\section{Participant Characteristics}

A total of 135 patients with CSDH were enrolled in the study. Thirty-eight patients were excluded from the study $(28.15 \%)$ (Figure 1). Among them, 43 patients received oral atorvastatin after the operation. There were no significant differences in the clinical or radiological characteristics between the atorvastatin group and the control group.

\section{Risk Factors for CSDH Recurrence}

The non-recurrent and recurrent patients in the control group differed significantly in terms of the $\mathrm{HB}[176.24 \pm 16.43$ vs. $194.25 \pm 12.34(\mathrm{~g} / \mathrm{L}), p<0.01], \mathrm{CT}$ value $[41.92 \pm 10.76$ vs. $34.12 \pm 8.78(\mathrm{Hu}), p<0.01]$, and low-density time $[3.88 \pm 1.04$ vs. $5.50 \pm 0.87$ (d), $p<0.01$ ] (Table 2). The non-recurrent and recurrent patients in the atorvastatin group differed significantly 

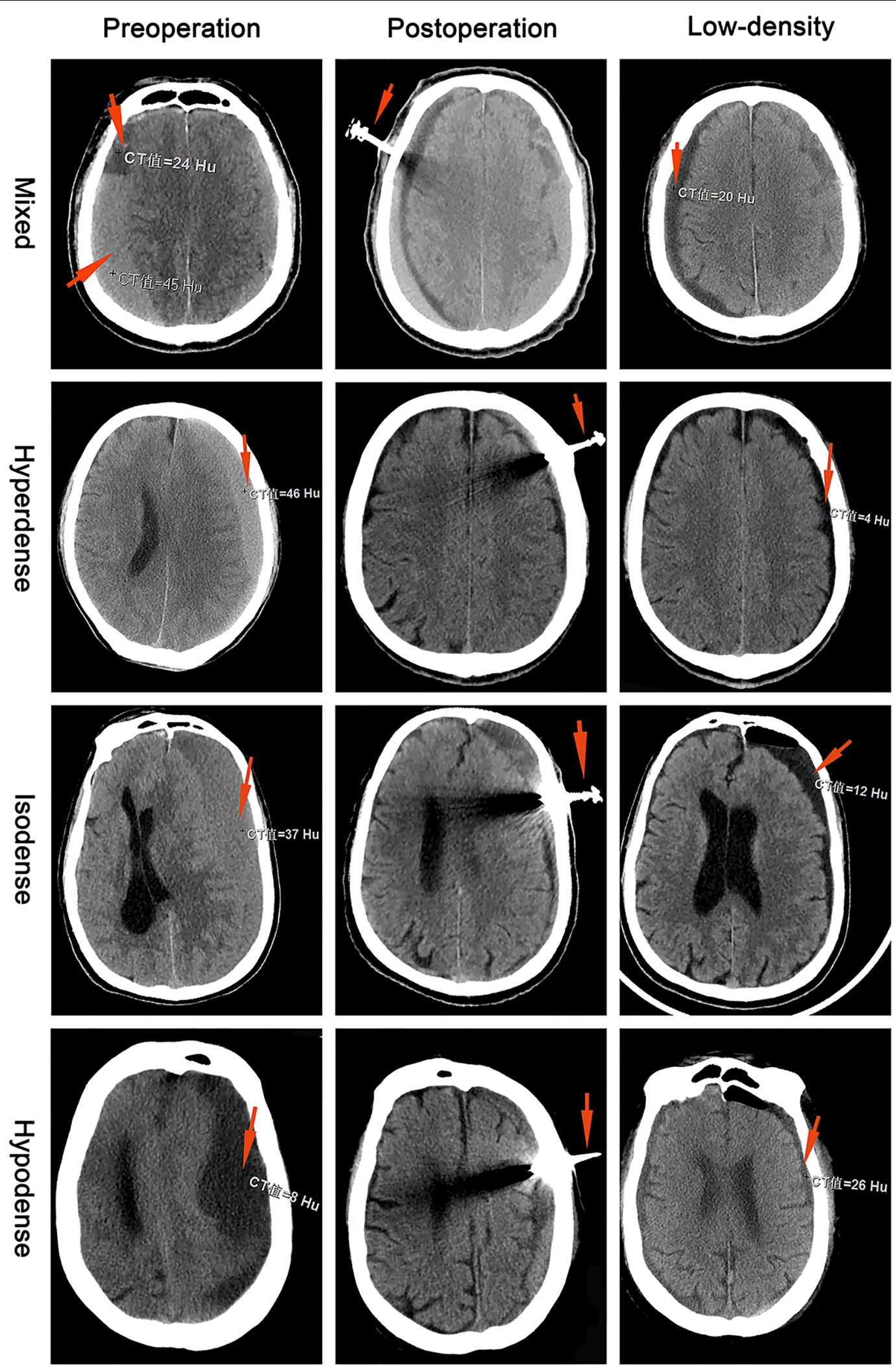

FIGURE 3 | Chronic subdural hematoma classification: mixed, hyperdense, isodense, and hypodense. The preoperative red arrows show the CT values (Hu) for different CSDH types. The postoperative red arrow shows the YL-1 drainage tube. The low-density red arrow shows that the CT value (Hu) of the CSDH was less than 30 . 


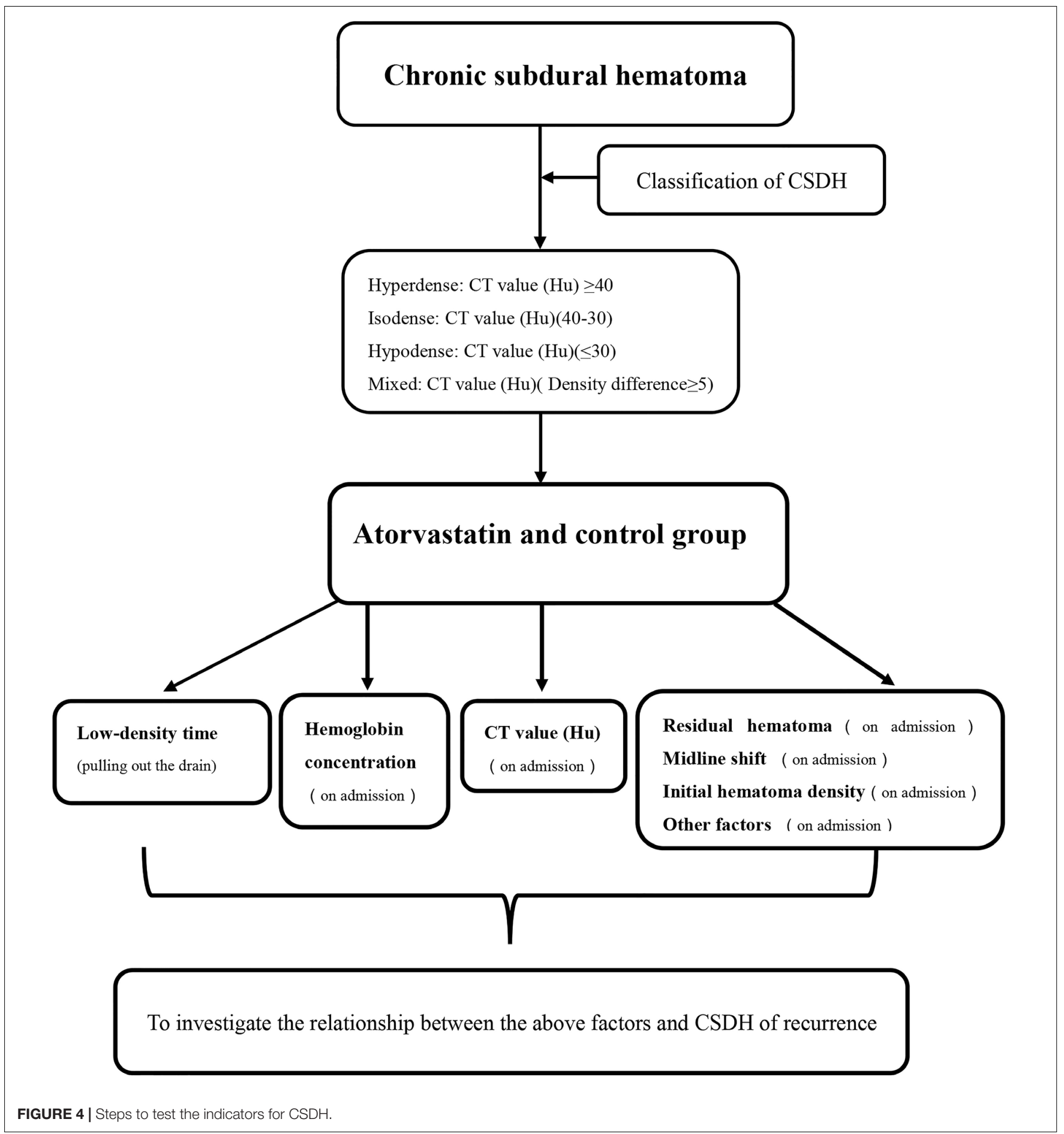

in terms of the $\mathrm{HB}[172.66 \pm 16.41$ vs. $190.45 \pm 10.23(\mathrm{~g} / \mathrm{L})$, $p<0.01]$, CT value $[38.91 \pm 7.16$ vs. $29.50 \pm 8.61(\mathrm{Hu})$, $p<0.01$ ], and mixed [2 vs. 4 (n), $p<0.05$ ] and low-density time [ $4.09 \pm 0.75$ vs. $5.45 \pm 1.12(\mathrm{~d}), p<0.01]$ (Table 2). The logistic regression analysis showed the comparison of the $\mathrm{HB}$ [odds ratio, 1.138; 95\% confidence interval (CI), 1.038-1.247], CT value $(\mathrm{Hu})$ (odds ratio, 1.127; 95\% CI, 0.995-1.277), and low-density time (odds ratio, 3.527; 95\% CI, 1.420-8.735) in the control group (Table 3). The HB (odds ratio, 1.126; 95\% $\mathrm{CI}, 1.029-1.233$ ), CT value $(\mathrm{Hu})$ (odds ratio, 1.062; 95\% CI, $0.975-1.156$ ), and low-density time (odds ratio, 2.259; 95\% CI, 1.103-5.799) were compared in the atorvastatin group (Table 3 ). The $\mathrm{HB}$ and low-density time might be risk factors for the recurrence of $\mathrm{CSDH}$. The ROC curves were used to measure the $\mathrm{HB}, \mathrm{CT}$ value $(\mathrm{Hu})$, and low-density time for predicting the cutoff value of recurrence after the operation. Specificity 
TABLE 1 | Simplified GCS and MGSS for the neurological conditions of the patients with $\mathrm{CSDH}$.

\begin{tabular}{|c|c|c|}
\hline GCS & MGSS & Symptoms \\
\hline 15 & 0 & No neurological symptoms \\
\hline $14-12$ & 1 & $\begin{array}{l}\text { Mild symptoms such as dizziness and headache, mild } \\
\text { neurological disorders such as asymmetric tendon reflexes }\end{array}$ \\
\hline $11-9$ & 2 & $\begin{array}{l}\text { Lethargy, misorientation, severe neurological impairment } \\
\text { such as mild hemiplegia }\end{array}$ \\
\hline 8-6 & 3 & $\begin{array}{l}\text { Shallow coma with appropriate response to pain stimuli, } \\
\text { severe neurological dysfunction such as hemiplegia }\end{array}$ \\
\hline $5-3$ & 4 & $\begin{array}{l}\text { Coma with a lack of response to pain stimuli, decerebrate, } \\
\text { or decorticate posturing }\end{array}$ \\
\hline
\end{tabular}

and sensitivity were also used as measures of CSDH recurrence accuracy. Receiver operating characteristic curve analysis showed that the area under the ROC curve (AUC) values for the HB, CT value $(\mathrm{Hu})$, and low-density time were $0.812,0.702$, and 0.755
TABLE 3 | Results of logistic regression analysis indicating predictors of risk factors.

\begin{tabular}{lccccc}
\hline Group Indicators & B & BE & Wald & $\boldsymbol{P}$-value & OR $(\mathbf{9 5 \%}$ CI) \\
\hline HB & 0.129 & 0.047 & 7.635 & $<0.01$ & $1.138(1.038-1.247)$ \\
CT Value $(\mathrm{Hu})$ & 0.119 & 0.064 & 3.511 & $>0.05$ & $1.127(0.995-1.277)$ \\
Low-density time & 1.260 & 0.463 & 7.422 & $<0.01$ & $3.527(1.42-8.735)$ \\
HB & 0.119 & 0.046 & 6.601 & $<0.01$ & $1.126(1.029-1.233)$ \\
CT Value $(\mathrm{Hu})$ & 0.60 & 0.44 & 1.904 & $>0.05$ & $1.062(0.975-1.156)$ \\
Low-density time & 0.928 & 0.423 & 4.800 & $<0.05$ & $2.529(1.103-5.799)$ \\
\hline
\end{tabular}

for all subjects (Table 4 and Figure 5A); 0.812, 0.719, and 0.790 for the control group (Table 4 and Figure 5B); and 0.807, 0.682, and 0.756 for the atorvastatin group (Table 4 and Figure 5C), respectively. The specificity and sensitivity for $\mathrm{HB}$ were higher than those for the CT value (Hu) and low-density time (Table 4 and Figures 5A-C).

TABLE 2 | Characteristics of patients with CSDH included in the control and atorvastatin groups.

\begin{tabular}{|c|c|c|c|c|c|c|}
\hline & \multicolumn{2}{|c|}{ Control group } & \multirow[b]{2}{*}{$p$ value } & \multicolumn{2}{|c|}{ Atorvastatin group } & \multirow[b]{2}{*}{$p$ value } \\
\hline & No recurrence $(n=42)$ & Recurrence $(n=12)$ & & No recurrence $(n=32)$ & Recurrence $(n=11)$ & \\
\hline Age $(y)$ & $60.00 \pm 13.66$ & $61.14 \pm 16.36$ & $p>0.05$ & $64.25 \pm 12.71$ & $62.82 \pm 13.51$ & $p>0.05$ \\
\hline Female sex & 7 & 2 & $p>0.05$ & 7 & 3 & $p>0.05$ \\
\hline Altitude & $2939.00 \pm 388.29$ & $2986.82 \pm 366.74$ & $p>0.05$ & $3085.69 \pm 343.66$ & $3049.33 \pm 389.29$ & $p>0.05$ \\
\hline Smoking & 10 & 3 & $p>0.05$ & 7 & 4 & $p>0.05$ \\
\hline Alcohol use & 13 & 3 & $p>0.05$ & 10 & 5 & $p>0.05$ \\
\hline \multicolumn{7}{|l|}{ Comorbidities } \\
\hline Diabetes $(n)^{\star}$ & 5 & 1 & $p>0.05$ & 3 & 1 & $p>0.05$ \\
\hline Hypertensio n (n) & 8 & 3 & $p>0.05$ & 8 & 4 & $p>0.05$ \\
\hline Heart disease $(n)^{\star}$ & 5 & 0 & $p>0.05$ & 2 & 0 & $p>0.05$ \\
\hline Pulmonary disease $(n)^{\star}$ & 1 & 1 & $p>0.05$ & 2 & 1 & $p>0.05$ \\
\hline Other diseases (n) & 7 & 2 & $p>0.05$ & 6 & 1 & $p>0.05$ \\
\hline \multicolumn{7}{|l|}{ Drug history } \\
\hline Antiplatelet treatment, No & 6 & 1 & $p>0.05$ & 5 & 1 & $p>0.05$ \\
\hline Antiplatelet treatment, $\mathrm{No}^{*}$ & 3 & 0 & $p>0.05$ & 3 & 0 & $p>0.05$ \\
\hline \multicolumn{7}{|l|}{ MGSS (Upon admission) } \\
\hline $\mathrm{M}_{0}$ & 0 & 0 & $p>0.05$ & 0 & 0 & $p>0.05$ \\
\hline$M_{1}$ & 20 & 5 & $p>0.05$ & 18 & 3 & $p>0.05$ \\
\hline $\mathrm{M}_{2}$ & 22 & 7 & $p>0.05$ & 15 & 7 & $p>0.05$ \\
\hline \multicolumn{7}{|l|}{ GCS (Upon admission) } \\
\hline $9(n)^{*}$ & 1 & 0 & $p>0.05$ & 0 & 1 & $p>0.05$ \\
\hline $10-12(n)^{*}$ & 2 & 1 & $p>0.05$ & 4 & 1 & $p>0.05$ \\
\hline $13-15(n)$ & 39 & 11 & $p>0.05$ & 28 & 9 & $p>0.05$ \\
\hline Platelet $\left(\times 10^{9}\right)$ & $212.79 \pm 54.06$ & $216.92 \pm 82.02$ & $p>0.05$ & $232.46 \pm 69.06$ & $209.34 \pm 55.51$ & $p>0.05$ \\
\hline Blood glucose (mmol/L) & $6.03 \pm 2.59$ & $6.30 \pm 0.93$ & $p>0.05$ & $6.22 \pm 1.74$ & $5.96 \pm 1.19$ & $p>0.05$ \\
\hline $\mathrm{HB}(\mathrm{g} / \mathrm{L})$ & $176.24 \pm 16.43$ & $194.25 \pm 12.34$ & $p<0.01$ & $172.66 \pm 16.41$ & $190.45 \pm 10.23$ & $p<0.01$ \\
\hline CSDH volume (ml) & $87.82 \pm 48.07$ & $91.75 \pm 40.15$ & $p>0.05$ & $84.73 \pm 56.32$ & $91.57 \pm 35.21$ & $p>0.05$ \\
\hline CT value $(\mathrm{Hu})$ & $41.92 \pm 10.76$ & $34.12 \pm 8.78$ & $p<0.01$ & $38.91 \pm 7.16$ & $29.50 \pm 8.61$ & $p<0.01$ \\
\hline \multicolumn{7}{|l|}{ Initial hematoma density } \\
\hline Hypodense $(n)^{\star}$ & 10 & 2 & $p>0.05$ & 7 & 3 & $p>0.05$ \\
\hline Isodense $(n)^{\star}$ & 17 & 3 & $p>0.05$ & 17 & 2 & $p>0.05$ \\
\hline Hyperdense $(n)^{\star}$ & 9 & 3 & $p>0.05$ & 6 & 2 & $p>0.05$ \\
\hline Mixed $(n)^{\star}$ & 6 & 4 & $p>0.05$ & 2 & 4 & $p<0.05$ \\
\hline \multicolumn{7}{|l|}{ Initial hematoma laterality } \\
\hline (bilateral: unilateral) $^{\star}$ & $6: 36$ & $3: 9$ & $p>0.05$ & $6: 26$ & $2: 9$ & $p>0.05$ \\
\hline Midline shift $\geq 10$ mm (n) & 14 & 5 & $p>0.05$ & 12 & 4 & $p>0.05$ \\
\hline Low-density time (d) & $3.88 \pm 1.04$ & $5.50 \pm 0.87$ & $p<0.01$ & $4.09 \pm 0.75$ & $5.45 \pm 1.12$ & $p<0.01$ \\
\hline Residual hematoma & 10.73 & 10.27 & $p>0.05$ & 12.61 & 12.32 & $p>0.05$ \\
\hline
\end{tabular}

${ }^{\star}$ Fisher. 


\section{Postoperative Follow-Up Results}

Table 5 summarizes the outcomes in the control and atorvastatin groups. The recurrence rate $(22.22 \%)$ found in the control group was significantly lower than the recurrence rate $(25.58 \%)$ found in the atorvastatin group $(P<0.05)$ (Table 5). Chronic subdural hematoma recurrence was observed in 12 patients in the control group and 11 patients in the atorvastatin group (Table 5). The number of patients with recurrence after the operation was six in the control group and four in the atorvastatin group (Table 5). Poor effects were found in five patients in the control group and five patients in the atorvastatin group (Table 5). Poor surgical result was found in three patients in the control group and two patients in the atorvastatin group (Table 5). There was no significant difference in the other evaluations (Table 5). There was no difference in the CSDH volume or MGSS between the control and atorvastatin groups on day 1 , months 1,2 , and 3 after the operation $(p>0.05)$ (Table 5).

\section{DISCUSSION}

This study was a retrospective study on the effect of atorvastatin on $\mathrm{CSDH}$ after burr-hole drainage at high altitude. The results showed that atorvastatin had no significant effect on hematoma absorption or on reducing the recurrence rate. It was found that the $\mathrm{HB}$ upon admission and the time for the hematoma to achieve a low density after the operation might have affected hematoma recurrence. However, because of the limited caseload, it may affect our conclusions. Burr-hole drainage is the first choice of treatment because of its limited amount of trauma, but the recurrence rate of hematoma is high. Studies have shown that atorvastatin can accelerate the absorption of postoperative CSDH and reduce the recurrence rate (Liu et al., 2016). Because of the special hypoxic environment, the $\mathrm{HB}$ increases at high altitudes. Hypoxia leads to brain inflammation (Zhou et al., 2017). Chronic subdural hematoma itself is a vascular response caused by chronic inflammation (Li et al., 2014). It has been speculated that the incidence of $\mathrm{CSDH}$ should be relatively high at high altitudes. In addition, brain injury is more serious, and neurological recovery is poor at high altitudes (Yu et al., 2014; Zhu et al., 2015; Wei et al., 2016, 2020). However, while we could not evaluate the prognosis of $\mathrm{CSDH}$ at high altitudes compared with low altitudes, our analysis showed that there was no difference in the CSDH volume or MGSS between the control group and the atorvastatin group on 1 day, 1, 2, and 3 months after the operation. What caused the poor therapeutic effect of atorvastatin calcium on CSDH at

TABLE 4 | Indices of lowest relative factors for predicting recurrence.

\begin{tabular}{|c|c|c|c|c|c|c|c|c|c|}
\hline & \multicolumn{3}{|c|}{ All subjects $(n=97)$} & \multicolumn{3}{|c|}{ Control group $(n=54)$} & \multicolumn{3}{|c|}{ Atorvastatin group $(n=43)$} \\
\hline & HB & CT value $(\mathrm{Hu})$ & $\begin{array}{l}\text { Low-density } \\
\text { time }\end{array}$ & HB & CT value $(\mathrm{Hu})$ & $\begin{array}{c}\text { Low-density } \\
\text { time }\end{array}$ & HB & CT value $(\mathrm{Hu})$ & $\begin{array}{c}\text { Low-density } \\
\text { time }\end{array}$ \\
\hline Cut-off value (\%) & $52.3 \%$ & $38.2 \%$ & $44.2 \%$ & $59.5 \%$ & $40.5 \%$ & $48.9 \%$ & $51.1 \%$ & $35.5 \%$ & $38.4 \%$ \\
\hline$A \cup C$ & 0.812 & 0.702 & 0.755 & 0.812 & 0.719 & 0.79 & 0.807 & 0.682 & 0.756 \\
\hline $95 \% \mathrm{Cl}$ & $(0.720-0.905)$ & $(0.569-0.835)$ & $\begin{array}{c}(0.668- \\
0.882)\end{array}$ & $(0.684-0.941)$ & $(0.540-0.898)$ & $\begin{array}{c}(0.650- \\
0.929)\end{array}$ & $(0.662-0.952)$ & $(0.481-0.883)$ & $\begin{array}{c}(0.568- \\
0.925)\end{array}$ \\
\hline Sensitivity (\%) & $73.9 \%$ & $65.2 \%$ & $73.9 \%$ & $83.3 \%$ & $66.7 \%$ & $75 \%$ & $72.7 \%$ & $63.6 \%$ & $72.7 \%$ \\
\hline Specificity (\%) & $78.4 \%$ & $73 \%$ & $72.1 \%$ & $76.2 \%$ & $73.8 \%$ & $73.8 \%$ & $62.5 \%$ & $71.9 \%$ & $65.6 \%$ \\
\hline$P$-value & $<0.01$ & $>0.05$ & $<0.05$ & $<0.01$ & $<0.05$ & $<0.01$ & $<0.01$ & $>0.05$ & $<0.05$ \\
\hline
\end{tabular}
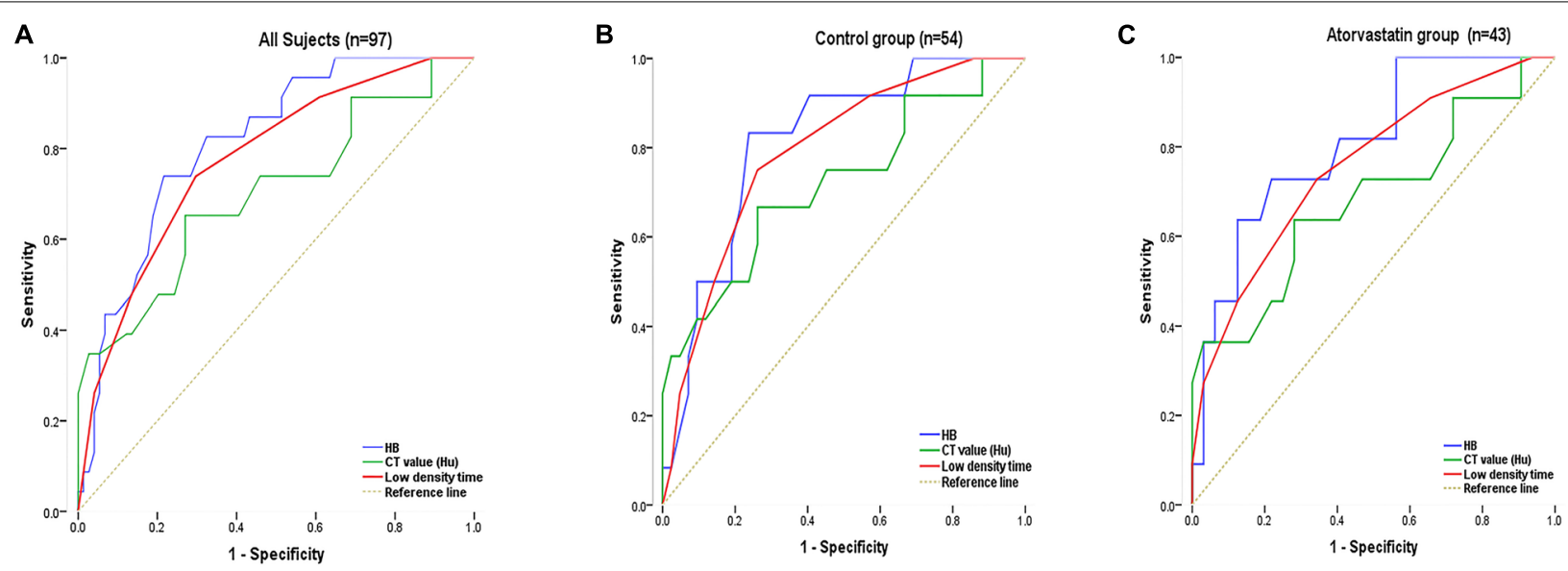

FIGURE 5 | The ROC curve was used to measure the HB, CT value (Hu), and low-density time for predicting the cutoff value of recurrence after surgery. Specificity and sensitivity were used as measures of $\mathrm{CSDH}$ recurrence accuracy. Analyses were performed for all subjects in the sample $(\mathbf{A} ; n=97)$ and separately for the control (B; $n=54)$ and atorvastatin $(\mathbf{C} ; n=43)$ groups. Chronic subdural hematoma classification recurrence was estimated using the AUC (A-C). 
TABLE 5 | Follow-up effect analysis in the control and atorvastatin groups.

\begin{tabular}{|c|c|c|c|}
\hline & $\begin{array}{c}\text { Control group } \\
n=54\end{array}$ & $\begin{array}{l}\text { Atorvastatin } \\
\text { group } n=43\end{array}$ & \\
\hline \multicolumn{4}{|l|}{ Non-recurrence } \\
\hline Poor effect & 5 & 5 & $>0.05$ \\
\hline Good effect & 24 & 25 & $>0.05$ \\
\hline Recurrence, No. (\%) & $12(22.22 \%$ & $11(25.58 \%)$ & $>0.05$ \\
\hline Recurrence with operation* & 6 & 4 & $>0.05$ \\
\hline Poor surgical result & 3 & 2 & $>0.05$ \\
\hline Time to recurrence & & & $>0.05$ \\
\hline 1 month & 9 & 10 & $>0.05$ \\
\hline 2 months ${ }^{*}$ & 2 & 1 & $>0.05$ \\
\hline 3 months ${ }^{*}$ & 1 & 0 & $>0.05$ \\
\hline \multicolumn{4}{|l|}{ CSDH volume (ML) } \\
\hline OV & $45.44 \pm 5.65$ & $43.12 \pm 7.89$ & $>0.05$ \\
\hline $1 \mathrm{~V}$ & $25.72 \pm 3.45$ & $23.64 \pm 5.42$ & $>0.05$ \\
\hline $2 \mathrm{~V}$ & $18.12 \pm 2.53$ & $19.41 \pm 3.11$ & $>0.05$ \\
\hline $3 \vee$ & $15.22 \pm 3.23$ & $15.57 \pm 2.32$ & $>0.05$ \\
\hline \multicolumn{4}{|l|}{ MGSS } \\
\hline $\mathrm{OM}_{0-1}$ & 30 & 28 & $>0.05$ \\
\hline $\mathrm{OM}_{2}$ & 23 & 15 & $>0.05$ \\
\hline $\mathrm{OM}_{3-4^{*}}$ & 1 & 0 & $>0.05$ \\
\hline $1 \mathrm{M}_{0-1}$ & 41 & 35 & $>0.05$ \\
\hline $1 \mathrm{M}_{2}$ & 13 & 9 & $>0.05$ \\
\hline $1 \mathrm{M}_{3-4}$ & 0 & 0 & $>0.05$ \\
\hline $2 \mathrm{M}_{0-1}$ & 46 & 38 & $>0.05$ \\
\hline $2 \mathrm{M}_{2}$ & 9 & 6 & $>0.05$ \\
\hline $2 \mathrm{M}_{3-4}$ & 0 & 0 & $>0.05$ \\
\hline $3 \mathrm{M}_{0-1}$ & 51 & 41 & $>0.05$ \\
\hline $3 \mathrm{M}_{2}^{*}$ & 3 & 2 & $>0.05$ \\
\hline $3 \mathrm{M}_{3-4}$ & 0 & 0 & $>0.05$ \\
\hline
\end{tabular}

$0,1,2$, and $3 \mathrm{~V}$ represent the $\mathrm{CSDH}$ volume on the first day, months 1,2 , and 3 after surgery, respectively. 0, 1,2, and $3 \mathrm{M}$ represent the MGSS on the first day, months 1, 2, and 3 after surgery, respectively. Low-density time, Time for CSDH to achieve low density (CT value $\leq 30)$. ${ }^{*}$ Fisher test.

high altitude? The result might be because of the special hypoxic environment or high $\mathrm{HB}$, which might affect the absorption of atorvastatin in hematoma. The findings might also be due to the fact that, with CSDH, the chronic inflammation of blood vessels is very serious, and the effect of atorvastatin calcium on the inhibition of the vascular response is poor. In addition, we retrospectively analyzed the risk factors for $\mathrm{CSDH}$ recurrence. The results showed that the risk factors for recurrence were $\mathrm{HB}$ and low-density time. We speculated that the higher the $\mathrm{HB}$ was, and the greater the hematoma inflammatory response, the more serious the vascular response around the hematoma cavity would be, and the greater the possibility of repeated bleeding. The longer the hematoma was absorbed below a CT value of 30, the higher the recurrence rate was, which might be due to the large inflammatory reaction of its own blood vessels and the possibility of repeated exudation. The longer the hematoma density was low [CT value $(\mathrm{Hu}) \leq 30$ ], the greater the chance of recurrence. At the same time, ROC curve analysis showed that the HB had high specificity and sensitivity. The ROC curve showed that the lowdensity time had a certain sensitivity for recurrence, but it was less sensitive than the HB. The ROC curve analysis was consistent with the logistic regression analysis: the $\mathrm{HB}$ and low-density time had statistical significance for hematoma recurrence. We analyzed the therapeutic effect of atorvastatin calcium on CSDH at high altitude. The results were not consistent with those at low altitude. However, we found some risk factors for recurrence from these limited case data, which might provide some methods for the future prevention of recurrence.

\section{CONCLUSION}

The effect of atorvastatin was not obvious after the operation. The risk factors for $\mathrm{CSDH}$ recurrence were $\mathrm{HB}$ and low-density time. The HB was the most specific and sensitive factor for predicting recurrence upon admission.

\section{Limitations}

This study was limited by the caseload and the retrospective design. These insufficiencies may limit the generalizability of our findings.

\section{DATA AVAILABILITY STATEMENT}

All datasets generated for this study are included in the article/supplementary material.

\section{ETHICS STATEMENT}

The studies involving human participants were reviewed and approved by the ethics committee of the Southwest Hospital. The patients/participants provided their written informed consent to participate in this study.

\section{AUTHOR CONTRIBUTIONS}

GZ designed this study. CL and LW collected the case data. MZ and JZ revised the manuscript to its final draft. LW contributed important discussion and interpretation of the results. All authors read and approved the final version of this manuscript.

\section{FUNDING}

This work was supported by Grant No. SWH2017JSZD07 to LW from the First Affiliated Hospital of Army Medical University "Science and Technology Innovation Program," Grant Nos. 81873754 and 81571116 to GZ from the National Natural Science Foundation of China.

\section{ACKNOWLEDGMENTS}

We acknowledge our colleagues (PLA 956th Hospital) for this study provided for this work. 


\section{REFERENCES}

Abouzari, M., Rashidi, A., Rezaii, J., Esfandiari, K., Asadollahi, M., Aleali, H., et al. (2007). The role of postoperative patient posture in the recurrence of traumatic chronic subdural hematoma after burr-hole surgery. Neurosurgery 61, 794-797. doi: 10.1227/01.NEU.0000298908.94129.67

Hanell, A., Hedin, J., Clausen, F., and Marklund, N. (2012). Facilitated assessment of tissue loss following traumatic brain injury. Front. Neurol. 3:29. doi: 10.3389/ fneur.2012.00029

He, C., Xia, P., Xu, J., Chen, L., and Zhang, Q. (2020). Evaluation of the efficacy of atorvastatin in the treatment for chronic subdural hematoma: a meta-analysis. Neurosurg. Rev. doi: 10.1007/s10143-019-01218-w [Online ahead of print]

Jaiswal, S. R., and Sontakke, S. D. (2012). Experimental evaluation of analgesic and anti-inflammatory activity of simvastatin and atorvastatin. Indian J. Pharmacol. 44, 475-479. doi: 10.4103/0253-7613.99311

Jang, K. M., Kwon, J. T., Hwang, S. N., Park, Y. S., and Nam, T. K. (2015). Comparison of the outcomes and recurrence with three surgical techniques for chronic Subdural Hematoma: single, double burr hole, and double burr hole drainage with irrigation. Korean J. Neurotrauma 11, 75-80. doi: 10.13004/kjnt. 2015.11.2.75

Khan, H. U., Atif, K., and Boghsani, G. T. (2019). Single versus double burr-hole drainage for chronic subdural hematoma: a study of relevant prognostic factors conducted in Pakistan. Pak. J. Med. Sci. 35, 963-968. doi: 10.12669/pjms.35. 4.543

Kim, D. H., Kim, H. S., Choi, H. J., Han, I. H., Cho, W. H., and Nam, K. H. (2014). Recurrence of the chronic subdural hematoma after burr-hole drainage with or without intraoperative saline irrigation. Korean J. Neurotrauma 10, 101-105. doi: 10.13004/kjnt.2014.10.2.101

Li, T., Wang, D., Tian, Y., Yu, H., Wang, Y., Quan, W., et al. (2014). Effects of atorvastatin on the inflammation regulation and elimination of subdural hematoma in rats. J. Neurol. Sci. 341, 88-96. doi: 10.1016/j.jns.2014.04.009

Liu, H., Luo, Z., Liu, Z., Yang, J., and Kan, S. (2016). Atorvastatin may attenuate recurrence of chronic subdural hematoma. Front. Neurosci. 10:303. doi: 10. 3389/fnins.2016.00303

Quan, W., Zhang, Z., Li, P., Tian, Q., Huang, J., Qian, Y., et al. (2019). Role of Regulatory $\mathrm{T}$ cells in Atorvastatin induced absorption of chronic subdural hematoma in rats. Aging Dis. 10, 992-1002. doi: 10.14336/AD.2018. 0926

Sahyouni, R., Goshtasbi, K., Mahmoodi, A., Tran, D. K., and Chen, J. W. (2017). Chronic subdural hematoma: a historical and clinical perspective. World Neurosurg. 108, 948-953. doi: 10.1016/j.wneu.2017.09.064

Soleman, J., Lutz, K., Schaedelin, S., Kamenova, M., Guzman, R., Mariani, L., et al. (2019). Subperiosteal vs subdural drain After burr-hole drainage of chronic subdural hematoma: a randomized clinical trial (cSDH-Drain-Trial). Neurosurgery 85, E825-E834. doi: 10.1093/neuros/nyz095

Tang, R., Shi, J., Li, X., Zou, Y., Wang, L., Chen, Y., et al. (2018). Effects of atorvastatin on surgical treatments of chronic Subdural Hematoma. World Neurosurg. 117, e425-e429. doi: 10.1016/j.wneu.2018.06.047

Wang, D., Li, T., Tian, Y., Wang, S., Jin, C., Wei, H., et al. (2014). Effects of atorvastatin on chronic subdural hematoma: a preliminary report from three medical centers. J. Neurol. Sci. 336, 237-242. doi: 10.1016/j.jns.2013.11.005

Wei, L., Chen, Z., Xi, Q., Wen, C., Ye, D., Chen, X., et al. (2016). Elevated Hemoglobin Concentration Affects Acute Severe Head Trauma After Recovery from Surgery of Neurologic Function in the Tibetan Plateau. World Neurosurg. 86, 181-185. doi: 10.1016/j.wneu.2015.09.070

Wei, L., Zhang, J., Zhang, B., Geng, J., Tan, Q., Wang, L., et al. (2020). Complement C3 participates in the function and mechanism of traumatic brain injury at simulated high altitude. Brain Res. 1726:146423. doi: 10.1016/j.brainres.2019. 146423

Xie, S. W., Liu, C., Gao, Y. X., Chen, Y., Jiang, C. H., Chen, L., et al. (2015). The study of prevalence rate, and clinical characteristics of high altitude deterioration. Eur. Rev. Med. Pharmacol. Sci. 19, 3444-3449.

Yu, A. Y., Xu, Q. H., Hu, S. L., Li, F., Chen, Y. J., Yin, Y., et al. (2014). Characteristics of a rat model of an open craniocerebral injury at simulated high altitude. Neuroreport 25, 1272-1280. doi: 10.1097/WNR.0000000000000259

Zhang, Y., Yang, Y., Long, S., and Li, G. (2020). Assessment of peripheral blood cell inflammatory markers in patients with chronic subdural hematoma. Clin. Neurol. Neurosurg. 191:105738. doi: 10.1016/j.clineuro.2020.105738

Zhou, Y., Huang, X., Zhao, T., Qiao, M., Zhao, X., Zhao, M., et al. (2017). Hypoxia augments LPS-induced inflammation and triggers high altitude cerebral edema in mice. Brain Behav. Immun. 64, 266-275. doi: 10.1016/j.bbi.2017.04.013

Zhu, H. T., Bian, C., Yuan, J. C., Liao, X. J., Liu, W., Zhu, G., et al. (2015). Hyperbaric oxygen therapy ameliorates acute brain injury after porcine intracerebral hemorrhage at high altitude. Crit. Care 19:255. doi: 10.1186/ s13054-015-097-978

Conflict of Interest: The authors declare that the research was conducted in the absence of any commercial or financial relationships that could be construed as a potential conflict of interest.

Copyright (c) $2020 \mathrm{Wei}$, Lin, Zhong, Zhang and Zhu. This is an open-access article distributed under the terms of the Creative Commons Attribution License (CC BY). The use, distribution or reproduction in other forums is permitted, provided the original author(s) and the copyright owner(s) are credited and that the original publication in this journal is cited, in accordance with accepted academic practice. No use, distribution or reproduction is permitted which does not comply with these terms. 